\title{
Recent Advances in Dental Sensors
}

\author{
Aastha Baldodia, Rajinder Kumar Sharma, Shikha Tewari, Aman Arora, and Muskan Baldodia
}

\section{ABSTRACT}

There have been advancements in sensing technologies in dentistry. Clinical applications in dentistry including Diagnosis as well monitoring disease and health in dental tissues are made easy with the advanced technologies in imaging and sensing tools. These recent advances in optical sources and detectors have unique applications including non-destructive testing, high speed and cost-effective electronic circuits, and novel signal processing methods. Some of the recent advances include optical coherence tomography, occlusal stabilization splint with stress-sensor system for bruxism, periodontal probe based on the fluorescent fiber position sensor, dental implantable temperature sensor for real time diagnosis of infectious periodontal disease, diamond probe/ perio2000 system, dental pulse oximeter with customized sensor holder, periodontal probe based on the flouroscent fiber position sensor etc.

Keywords: Advancements, caries, periodontal, sensors.
Published Online: January 7, 2022

ISSN: $2684-4443$

DOI: 10.24018 /ejdent.2022.3.1.127

\author{
A. Baldodia* \\ Postgraduate Institute of Dental \\ Sciences, Rohtak, India. \\ (e-mail: aasthabaldodia626@gmail.com) \\ R. K. Sharma \\ Postgraduate Institute of Dental Sciences, \\ Rohtak, India. \\ (e-mail: rksharmamds@yahoo.in) \\ S. Tewari \\ Postgraduate Institute of Dental Sciences, \\ Rohtak, India. \\ (e-mail: drshikhatewari@yahoo.com) \\ A. Arora \\ Dr. Aman Arora's Dental Care- Braces \& \\ Implants, Faridabad, India. \\ (e-mail: amanarora8412@gmail.com) \\ M. Baldodia \\ Amity University, Noida, India. \\ *Corresponding Author
}

\section{INTRODUCTION}

There have been advancements in sensing technologies in dentistry. Clinical applications in dentistry including Diagnosis as well monitoring disease and health in dental tissues are made easy with the advanced technologies in imaging and sensing tools. These recent advances in optical sources and detectors have unique applications including non-destructive testing, high speed and cost-effective electronic circuits, and novel signal processing methods.

\section{DiscUSSION}

Following are the various advancements that we have witnessed in recent times

\section{A. Optical Coherence Tomography (OCT)}

Dental caries; an infectious polymicrobial disease of teeth causing demineralisation in a localised area of tooth. With the increase in oral fluoridated products used for anti-caries action, smooth surface caries has reduced consistently but there has been an increased rate in pit and fissure caries [1]. These hidden or occult caries pose difficulty in diagnosis making clinically. Visual examination along with radiographs is commonly used to analyze the demineralized lesion but these methods are insensitive for detection of carious lesion at an early stage. Hence, OCT has emerged as an advanced modality in providing images of internal structure in a non-invasive way [2].

A handheld scanning probe being placed at a stable point over the tooth, and the scanning beam was oriented ninety degrees to the occlusal surface. Its mechanism is based on recognition of the backscattered light produced by sub micrometer size blemishes made in demineralized lesions [3].

\section{1) Advantages}

1. It leads to a rise in signal strength which seems as bright areas in OCT images that is easily differentiated from adjacent sound enamel. Its ability to detect microstructural defects in the tooth is unique. It also detects other characteristics like the spatial features, such as the size of the cavitation and lateral spread of caries along the dentinoenamel junction.

2. The main advantage is that X-ray exposure is not required, and also enables real-time visualisation.

3. It can be considered as a time efficient and safe option for accurate diagnosis of caries extent.

\section{2) Limitations}

1. The visual interpretation of OCT images requires appropriate calibration.

2. The imaging depth range could be limited to $2-3 \mathrm{~mm}$ or shallower depending on the attenuation of the near infrared light. This modality may face limitations for deeper clinical applications as the intricate light scattering in dentin is also influenced by the composition and structural geometry of the substrate. 


\section{3) Suggestions}

1. A system program should be constructed for homogenizing calibrations and eliminating calibration errors.

2. Image depth range should be upgraded for improved resolution of images.

\section{B. Occlusal Stabilization Splint with Stress-Sensor System for Bruxism}

Bruxism is one of the chronic dental problems worldwide with multifactorial etiology and no golden standard for diagnosis and treatment [4]. This is defined as repetitive jaw muscle activity characterized by clenching or grinding of teeth. Current diagnostic methods mainly include self-report, clinical examination, electromyography, and polysomnography [5]. For the diagnosis as well as treatment of bruxism, a real time, intelligent, quantitative and precise force based biofeedback detection device based on artificial intelligence algorithm is invented. This sensor system is made up of the main control module, another module for acquiring pressure signals, and a server terminal module. A sandwich method or a layering technique was used to integrate stress sensing devices in an occlusion stabilization splint which is resin based. For further occlusal force data processing, and then configuring the parameters, a machine-based algorithm is used. They used a system prototype system to completely evaluate every part of the occlusal splint [6].

\section{1) Advantages}

1. Reasonable parametric components were found for the sensor system, which further showed feasibility for the proposed scheme for the treatment of bruxism.

2 . It demonstrates a promising method to diagnose bruxism and its treatment.

\section{2) Limitations}

1. These stress sensing chips may demonstrate deterioration under high temperature and stress.

2. They are still under investigation and are not available in the dental market.

\section{3) Suggestions}

1. The main problem lies in the effective collaboration of the traditional materials that require high temperature and stress fabrication with the chips that needed low temperature and stress, which must be solved first and foremost.

2. Clinical studies should be undertaken to evaluate the efficiency of the sensor system so that it can be released in the dental market in the coming future.

\section{Flexible Phosphor Sensors (POS)}

Most dental practices that have renewed to digitalisation utilize rigid or direct sensors. Another digital advancement that has occurred is the indirect sensors, or Phosphor storage plates (PSP) [7]. These are wireless, non-rigid, indirect digital sensors that do not connect directly to the computer. The image produced is stored in the PSP as a latent image. This PSP is then connected to the computer and the image is scanned and digitized by the PSP and eventually gets stored and eventually displayed in a few seconds on the computer [8].

\section{1) Advantages}

1. Added edge to certain patients who are aversive to direct sensors.
2. The sensor is smaller, thinner, and softer than rigid sensors. Therefore, conforms better to the anatomical structures in the oral cavity.

3. No bulky wires are not a matter of concern.

4. Paediatric, and elderly patient experience is more pleasant.

5. It put forward a bigger image area making the clinician measure the coronal to the apical length of the tooth more accurately.

6. Fewer retakes are needed thus less radiation exposure.

7. Cost is also an advantage with PSP over rigid sensors. Insurance is rendered unnecessary because there is no costly purchase and additionally because of the wireless nature, chances of accidental breakage are less.

8. There is no need for the staff to learn new protocol when upgrading from film radiography as it utilizes the exact workflow.

\section{2) Limitations}

1. Image processing may be faster than film but slower than with the direct sensors.

2. General practitioners who need to take radiographs constantly throughout the dental procedure may opt for rigid sensors due to instant image formation.

\section{3) Suggestions}

Ultra-compact, cost effective processors are needed to be designed for chairside use so that the image is appeared on the monitor in very little time.

\section{Periodontal Probe Based on the Flouroscent Fiber Position Sensor}

Periodontal diseases (gum diseases) are prevalent in developed as well as developing countries affecting almost 20-50 percent of global population [9]. This is a photonic smart probe based on fluorescent fiber. Clinical attachment loss (CAL) is the gold standard for evaluation and treatment planning of periodontitis.

In this device, the flouroscent linear optical fiber position sensor is adapted to a probe system specifically the second generation. A customized surgical calliper attached to the periodontal probe takes the measurements and converts the unit depth of probe which is in mm scale according to the excitation length of the florescent fiber. This resulted in $\mathrm{cm}$ scale reading [10].

\section{1) Advantages}

1. It will be cost-efficient.

2. It is quick to give accurate results.

3. It is comfortable to work with because of its lightweight nature.

4. It does not involve complex instructions, hence easy to use.

\section{2) Limitations}

These probes are not manufactured for the dental market which constitutes its major limitation

\section{3) Suggestions}

Clinical trials should be conducted to evaluate the efficiency and accuracy of this device in dental management of disease. 


\section{E. Diamond Probe/ Perio2000 System}

This probe is used to detect periodontal disease activity during routine dental examination [11]. It works by measuring the relative amount of sulphide concentration that acts as an indicator of gram-negative bacterial activity in pyorrhoea affected teeth.

A system is built which is composed of a disposable, single-use probe tip and a microsensor connected to a control unit.

\section{1) Advantages}

The basic advantage constitutes its ability to detect periodontitis at an early stage of activity and pinpoint an active site of disease that demanded intervention to halt its further progress to severe conditions

\section{2) Limitations}

1. The probing pressure is not controlled, so chances of false positive results are present.

2. Periodontal diseases are also caused by bacteria other than those producing volatile sulphide end products that possess the potential to miss disease activity in periodontally inflicted sites.

\section{3) Suggestions}

1. Other modifications should be incorporated in the probe including the addition of chemical sensing mechanisms so that other indicators of the periodontal disease activity along with volatile sulphur compounds can be monitored.

2. Clinical studies should be conducted to validate the results and efficiency of this probe for dental practice.

\section{F. Dental Pulse Oximeter with Customized Sensor Holder}

Various pulp tests are available in the dental market but are based on patient's subjective responses that questions the reliability of these tests. A pulse oximeter is a non- invasive device that is used to assess the vascular response of the tooth pulp and renders aid in root canal treatment of tooth [12]. The blood supply to the tooth pulp can be detected by the oxygen saturation levels of the pulp. This device is comprised of a pulse oximeter monitor, sensor holding probe, and a sensor holder. This is an objective test that acts as a reliable indicator for the assessment of correct status of tooth pulp [13.] For instance, there can be a possibility on the part of an apprehensive patient that he misinterprets and provides false positive or false negative response to the operator.

\section{1) Advantages}

1. Neonatal sensors were utilized, and appropriate custom made sensor holder was designed to help in accurate positioning and placement of the sensor on the tooth surface.

2. The sensor holding device has an added advantage in terms of effective positioning of the sensor for proper recording of pulpal status of the tooth. 3 dimensional printing method was used to design this flexible sensor holder which facilitates easy placement of the sensor on the tooth surfaces.

3. Pulse oximeter usage demonstrated highest diagnostic accuracy for evaluating pulpal status of the tooth. Receiver operating curve (ROC) originate from the left-handed border and concluded at the top border of the space. This is away from the 45 degree diagonal line. It indicates reliability in assessing the actual pulp status of the tooth.

\section{2) Limitations}

1. The main drawback is that these probes are not used in the dental practice because they are not yet to be manufactured for the dental market.

\section{3) Suggestions}

1. Clinical trials with an adequate sample size should be conducted to evaluate the accuracy and efficiency of these devices so that the process of their manufacturing is exalted for dental use.

2. These devices should be cost efficient and easy to use.

G. Dental Implantable Temperature Sensor for Real Time Diagnosis of Infectious Periodontal Disease

It is a polymer based flexible sensor that detects temperature differences for dental implant systems. Current diagnostic methods for diagnosing peri-implantitis are based on the clinical signs and symptoms of the implant supporting tissues due to disease activity [14]. These are subjective methods that lack precision also. These are time delayed as the time difference between the onsets and reporting of the symptoms to the dentist is highly variable. One successful solution to overcome this technical hurdle is to incorporate nano or micro technologies into the point of care (POC) devices [15]. These implantable or wearable devices have already been developed. At home care management these allow real time and continuous health monitoring. It has been shown in various studies that temperature has a direct correlation with systemic inflammation. In the past also, various implantable or wearable devices have been developed, and successfully utilized for monitoring in various organs including the cardiac tissue, etc.

\section{1) Advantages}

1. Various stress studies have indicated sufficient performance and high stability as well as good results in terms of repeatability also.

2. The sensor is compact and allows efficient onsite sensing having multiple channels around the implant.

3 . The design of the sensor is quite flexible.

4. Repeated calibration of the readings is not necessary. No initial calibration is necessary. As the baseline temperature monitoring is continuous and it works on the reproducible resistance temperature relationship.

5. The sensor is made up with most biocompatible materials and represents a constant TCR value throughout the testing along with no damage to the polymer film.

\section{2) Limitations}

1. Battery recharging is an issue.

2. Temperature change is the only parameter that is detected and analyzed.

3. These devices are yet to launch in the dental market.

\section{3) Suggestions}

1. It should be integrated to dental implant with wireless connection and battery recharging capabilities.

2. There should be simultaneous detection of other physio chemical mechanisms so that it can sense other multiple factors along with temperature change.

3. They should upgrade the sensor so that it can be embedded in the chairside set up and can be used efficiently for oral diagnosis. 


\section{FUNDING}

There is no source of funding.

\section{CONFLICT OF INTEREST}

Authors declare that they do not have any conflict of interest.

\section{REFERENCES}

[1] Desai V, Bumb D. Digital dental photography: a contemporary revolution. International journal of clinical pediatric dentistry. 2013 Sep;6(3):193.

[2] Amaechi BT, Higham SM, Podoleanu AG, Rogers JA, Jackson DA. Use of optical coherence tomography for assessment of dental caries: quantitative procedure. Journal of oral rehabilitation. 2001 Dec;28(12):1092-3

[3] .Luong MN, Shimada Y, Araki K, Yoshiyama M, Tagami J, Sadr A. Diagnosis of occlusal caries with dynamic slicing of $3 \mathrm{~d}$ optical coherence tomography images. Sensors. 2020 Jan;20(6):1659.

[4] Glaros AG, Rao SM. Bruxism: a critical review. Psychological Bulletin. 1977 Jul;84(4):767.

[5] Reddy SV, Kumar MP, Sravanthi D, Mohsin AH, Anuhya V. Bruxism: a literature review. Journal of international oral health: JIOH. 2014 Nov;6(6):105.

[6] Gao J, Liu L, Gao P, Zheng Y, Hou W, Wang J. Intelligent Occlusion Stabilization Splint with Stress-Sensor System for Bruxism Diagnosis and Treatment. Sensors. 2020 Jan;20(1):89.

[7] Glazer HS. Flexible phosphor sensors: a digital supplement or option to rigid sensors. Compendium of continuing education in dentistry (Jamesburg, NJ: 1995). 2014 Jul 1;35(7):512-6.

[8] Bhuyan SK, Rout RN. Dental Imaging Array Technologies: A Review and Update. Indian Journal of Mednodent and Allied Sciences. 2017;5(1):60-7.

[9] Nazir MA. Prevalence of periodontal disease, its association with systemic diseases and prevention. International journal of health sciences. 2017 Apr;11(2):72.

[10] Galatus RM, Marita T, Buzura L, Ilea A. Periodontal probe based on the fluorescent fiber position sensor. InBiophotonics in Point-of-Care 2020 Apr 1 (Vol. 11361, p. 113610W). International Society for Optics and Photonics.

[11] Zhou H, McCombs GB, Darby ML, Marinak K. Sulphur by-product: the relationship between volatile sulphur compounds and dental plaque-induced gingivitis. J Contemp Dent Pract. 2004 May 1;5(2):2739.

[12] Janani K, Palanivelu A, Sandhya R. Diagnostic accuracy of dental pulse oximeter with customized sensor holder, thermal test and electric pulp test for the evaluation of pulp vitality: an in vivo study. Brazilian dental science. 2020 Jan 31;23(1):8-p.

[13] Gopikrishna V, Tinagupta K, Kandaswamy D. Comparison of electrical, thermal, and pulse oximetry methods for assessing pulp vitality in recently traumatized teeth. Journal of endodontics. 2007 May 1;33(5):531-5.

[14] Ramanauskaite A, Juodzbalys G. Diagnostic principles of periimplantitis: a systematic review and guidelines for peri-implantitis diagnosis proposal. Journal of oral \& maxillofacial research. 2016 Jul;7(3).

[15] Kim JJ, Stafford GR, Beauchamp C, Kim S. Development of a Dental Implantable Temperature Sensor for Real-Time Diagnosis of Infectious Disease. Sensors. 2020 Jan;20(14):3953. 